\title{
ARTICLE
}

\section{Summary of Actinides 2017 - physics and materials science}

\author{
Gerry H. Lander ${ }^{*}$ \\ European Commission, Joint Research Centre, Directorate for Nuclear Safety and Security, Postfach 2340, D-76125 Karlsruhe, \\ Germany
}

The text below contains some of the remarks I made at the end of the Actinides 2017 Conference in Sendai, Japan, 10-14 July, 2017

Keywords: actinide; physics; materials science

\section{Introduction}

I shall start this short summary with a discussion of the basic physics behind the metals, the dioxides, and heavy-fermion systems. I shall then pass on to the much wider field of materials science, which dominated the Conference, at least in number of presentations. Since atomic and super-heavy element physics are beyond my competence, I cannot make any remarks on these subjects. However, I do admire techniques that can glean information from femtogram samples or even single atoms. I hope these fields stay a part of this Conference series, and that slowly we can find connections between this exotic field of study and the more mundane examples presented by the majority - they demonstrate the necessity of working on minute samples, which will be needed to extend work in the future on transuranium materials. Working with large samples of transuranium samples will become increasingly difficult, due to safety considerations, but I argue in the conclusions that we must preserve this capability even if the amounts get much smaller than presently allowed.

\section{Major physics questions}

\subsection{Plutonium metal}

Discovered in 1941, the puzzles of $\mathrm{Pu}$ were already evident in the Manhattan project. No theory could handle $\mathrm{Pu}$ until the mid-1970s, when the first band-structure attempts showed the great difficulties. Density functional theory provided a major advance in the 1980s but methods based on that formalism, such as $\mathrm{LDA}+U$ and $\mathrm{GGA}+U$, predict ordered magnetism due to the strong correlations when $55 f$ electrons are introduced. It was not until a landmark paper in Nature in 2007 [1], introducing the dynamical-mean field theory

*Corresponding author. Email: lander@ill.fr
(DMFT), that the resolution of this problem became possible. Predictions of the $5 f$ spectral function in this, and subsequent, papers showed that the $5 f$ states are mixed valent, and the $5 f$ states have a strong interaction with the conductions electrons in the metal - the so-called Kondo interaction.

Early efforts to observe the Kondo resonance by using neutron inelastic scattering at Los Alamos were not successful, but an experiment at the higher-intensity spallation neutron source at Oak Ridge was successful in

2013, and the paper published in 2015 [2].

Our understanding has been further advanced by a complete paper by Amadon [3] in 2016, and extension of the theory to include temperature effects by Dorado et al. [4], and Söderlind [5] - much of this presented at the Conference.

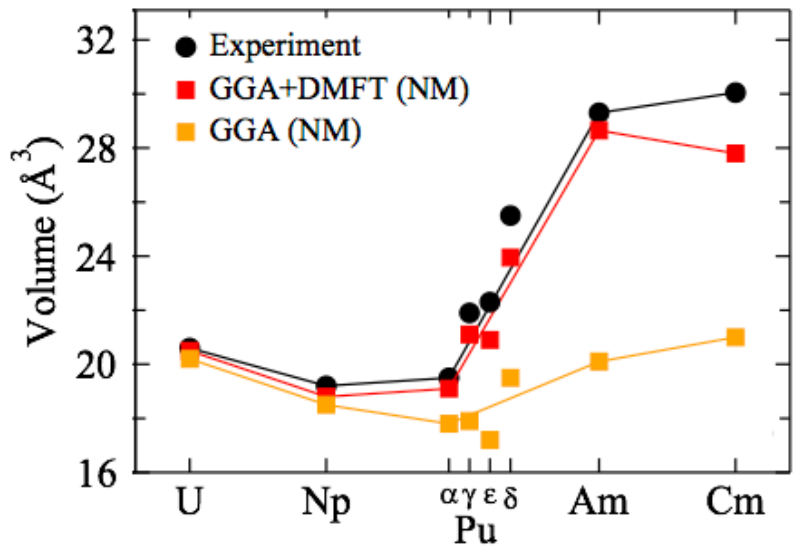

Figure 1. Volume as a function of atomic number as calculated with the various codes in the key - taken from B, Amadon [3]. NM signifies non-magnetic.

Figure 1 shows the recent work of Amadon [3] in which the correct volumes (and bulk moduli; not shown here) are obtained with no need for magnetic ordering. The latter is necessary if only the GGA method is used, 
as evident by the disagreement of the GGA (NM) calculation shown in the figure. This represents a significant advance in our understanding of the light actinides, and shows the importance of the DMFT methodology.

Although some details of the photoemission of $\mathrm{Pu}$ still remain unclear (and there is no ARPES data to test against theory as no sizeable single crystals of $\delta$-Pu have been produced) [6], the main outlines of our understanding of Pu metal are clearer now than for many years. One other major problem remaining is the understanding the sharp drop of the bulk modulus in $\delta$-Pu as it is heated, as measured by Migliori et al.[7].

\subsection{Uranium dioxide}

$\mathrm{UO}_{2}$ has been investigated since at least 1952, and yet Gofryk et al. (Idaho National Lab) gave us two examples of unexpected experimental effects observed over the last two years. The first is the observation of anisotropic thermal conductivity [8] in this system. Because $\mathrm{UO}_{2}$ is cubic this is forbidden by symmetry. The second is the observation at high magnetic fields $(\mathrm{H}$ $>15 \mathrm{~T}$ ) of an unusual piezomagnetic effect, in which strain is induced by the applied magnetic field. Both these observations may be related to effects related to the quadrupolar interactions, and complications therefrom, in $\mathrm{UO}_{2}$ [9]; and call for more experiments (and theory) to explain them.

Another contribution on $\mathrm{UO}_{2}$ was by $\mathrm{J}$. Tobin who reported synchrotron experiments showing that both $\mathrm{UF}_{4}$ and $\mathrm{UO}_{2}$ (both nominally $5 f^{2}$ systems), show $6 d$ covalency, (albeit small in $\mathrm{UF}_{4}$ ) but only $\mathrm{UO}_{2}$ involves also covalency with the $\mathrm{U} 5 f$ states.

\subsection{Heavy Fermions and superconductivity}

This subject has, of course, been present at Actinide Conferences for the last 30 years. As with high- $\mathrm{T}_{\mathrm{c}}$ materials, which now feature $\mathrm{Fe}$ as well as the historic $\mathrm{Cu}$ materials, there is still no accepted theory for explaining their superconductivity. The Conference featured a number of new findings. Particularly important were contributions by Aoki (Sendai) on ferromagnetic superconductors; Fujimori (Spring-8) on angular-resolved photoemission studies (ARPES); Kittake (Tokyo) on the specific heat as a function of the direction of the applied magnetic field; Caciuffo (EC, Karlsruhe) and Wilhelm (ESRF, Grenoble) on X-ray circular dichroism (XMCD); and Griveau (EC, Karlsruhe), on the importance of defects in characterizing the Pu superconductors. All these studies, often using techniques not previously applied to these materials, give new information on the ground states of the heavy-fermion superconductors. They thus increase the knowledge base of these fascinating systems, and provide further benchmarks for theory.

\section{Technique development}

Some relatively new techniques were already mentioned, such as ARPES and XMCD, but with the rapid development in synchrotron techniques actinide researchers have to stay abreast and be ready to request access to these facilities. For example, recently it has been possible to perform Resonant X-ray Emission Spectroscopy (RXES) at the actinide $M_{4,5}$ edges, as well as the already published work at the actinide $L_{2,3}$ edges by Booth et al. [10]. The $5 f$ electrons are probed more directly at the $M$ edges, as compared to the situation at the $L$ edges, so indeed the results are not exactly the same [11]. This technique can also be applied to more chemistry-related materials, and a good example is the recent study on actinyl ions by Vitova et al. [12].

A new technique, related to the one above, but now performed with soft X-rays ( $<1 \mathrm{keV}$ incident energy) on single crystals has been used in transition-metal systems, especially high- $\mathrm{T}_{\mathrm{c}}$, to measure elementary excitations and their dispersion across the Brillouin zone [13]. It is clear that understanding such experiments in actinides would lead to a better knowledge of the orbital bonding and covalency. So far, no experiments have been reported at the actinide $N$ edges, which lie at a very convenient energy of close to $700 \mathrm{eV}$.

\section{Materials science - the rest}

\subsection{Examples in materials science}

The vast majority of the presentations at Actinides 2017 outside of subjects already discussed above (or chemistry) can be classified in the area of materials science. Such studies cover a multitude of subjects, often directly related to real-life problems in the nuclear industry. Some of these are related to new fuels, and even new reactor types, but the bulk of the presentations were related to present-day problems of using oxide fuel. This is, of course, very commendable, especially given the huge challenges of cleaning up the Fukushima disaster in Japan, and the long-standing problems associated with early weapons production centers in many of the nuclear-weapons states.

Interesting presentations were made by Benes (ITU) on facilities for materials science work beyond uranium; Ravat (CEA-Valduc) on kinetics of $\mathrm{Pu}$ oxide growth; Delaunay (CEA-Valduc) on $\mathrm{H}_{2} \mathrm{O}$ adsorption and disassociation on oxidized $\mathrm{Pu}$ metal; Vinkurov (Russia) on Properties of highly active actinides in MPP $\mathrm{MgKPO}_{4} \cdot 6 \mathrm{H}_{2} \mathrm{O}$; and by Allen (LLNL) on radiation damage in $\mathrm{Pu}$ alloys.

I have picked these examples above because all of them address materials science problems by actually working on radioactive materials.

\subsection{The challenge to work with transuranium materials}

It goes without saying that the challenges of Fukushima and many other places are particularly 
significant because they involve highly radioactive materials, and sizeable quantities of plutonium, and other dangerous isotopes. Of course, working on irradiated fuel is especially difficult and requires hot-cell facilities that exist in fewer places today than they did in the 1960s. These are indeed very expensive and we can expect them to slowly close as the maintenance costs continue to rise. That is bad enough for our field, but in a sense worse, is the continuing closure of glove boxes and facilities that can be used for many other studies on transuranium materials.

I urge the participants at this Conference to regard transuranium facilities as part of the "heritage" of our field. These facilities are expensive and the burden of the ever-increasing "safety" bureaucracy means that they face an uncertain future. That is very bad news for all actinide researchers, even if your present efforts involve only uranium.

Two examples from this Conference are worth mentioning. First, a distinguished German chemist lamented during a plenary talk that there were no facilities for transuranium chemistry in Germany. That is unfortunate, as the statement is false, the European Commission's (EC) laboratory in Karlsruhe has those facilities, and they need to be supported by a far larger community. The EC, itself something of bureaucratic nightmare, is not easy to work with, but scientists in Europe need to push for more involvement in this laboratory. Second, in Japan, the OARAI facility, in which many transuranium materials have been made by the JAEA group at Tokai, is at risk of closure. Given the long-term challenges of the Fukushima disaster, this seems to me incomprehensible, and I urge all Japanese actinide researchers, whether they have been to OARAI or not, to try to prevent a loss of such capabilities.

\subsection{Lowering the radioactive inventory in actinide research}

It has been recognized for a number of years that if the radioactive inventory of samples can be lowered, a number of new systems can be examined without complicated new transuranium laboratories. The best examples of this are the use of thin films. Today, with sputtering, molecular-beam, and chemical vapor techniques extremely well-characterised (even single crystal or epitaxial) samples can be produced. Uranium samples of $\sim 10 \mathrm{~nm}$ thickness on a 10 x $10 \mathrm{~mm}^{2}$ substrate represent far less than $1 \mathrm{~Bq}$, so are of no danger whatsoever. Of course, for transuranium samples the samples have to be capped, but this is relatively easy. Gouder and colleagues used this technique for photoemission studies of $\mathrm{Pu}$ and compounds in-situ in Karlsruhe in a hot laboratory [14]; the thin samples guaranteed the absence of oxygen, but the samples were not removed from the sputtering system.

One of the first laboratories to exploit this technique was LANL with early work on $\mathrm{UO}_{2}$ and $\mathrm{U}_{3} \mathrm{O}_{8}$ [15]. More recently, they have made $\mathrm{PuO}_{2}$ epitaxial films. Ward at Oxford University was the first to produce epitaxial films of $\alpha-U[16,17]$, and this technology has now been adopted at Bristol University, where Springell and colleagues have undertaken a number of different studies in materials science reported at this meeting. New efforts with the thin film approach, some of which were reported at the meeting, include groups at Charles University, Prague on hydrides, and Tokai, Japan with interest in spintronics, and a group in China who reported thin-film production of Ce-La alloys, but will move soon to uranium. Another interesting approach using thin films to study radiation damage was reported from M. Shandalov and colleagues from the Nuclear Centre in Israel. I commend all these efforts and hope soon that they will be applied to transuranium systems, as the small quantities of active isotopes needed are a great advantage when considering a range of experiments. This is certainly a field that will grow.

The use of $3^{\text {rd }}$ generation synchrotron radiation is also a way to reduce the inventory. High-pressure studies, such as that of curium represent a good example, where the sample was only a few micro-grams. [18]. This is certainly an area where some of the expertise in heavy-element chemistry could be used; those researchers have a great shortage of material and have been forced to develop techniques suitable for nanograms.

\section{Conclusion}

The Conference in Sendai shows that actinide research is in good hands, with progress across a broad spectrum on both the fundamental and applied fields. Of course, given the disaster of Fukushima there is naturally a great tendency to push for applied work. One can understand and condone that, but the fundamental science is important in the long run. A good example is the improvement in the understanding of $\mathrm{Pu}$ metal, most of which has actually come from new theoretical tools, first worked out for the transition metals and then applied also to the actinides.

Sometimes in the "Actinide Conferences" I get the impression we are at a "Uranium Conference". Of course, it is much harder to work beyond $\mathrm{U}$, but we must always remember that this is crucial if we are indeed at an Actinide Conference. Talk of using $\mathrm{Ce}$ as a "surrogate for Pu" should be taken with much caution. Pu has many more valence states than $\mathrm{Ce}$, and, importantly, the most common isotope of $\mathrm{Pu}, 239$, damages itself overtime. One can understand an initial study using Ce, but the final experiments must move towards experiments with $\mathrm{Pu}$. Researchers must not lose sight of this.

\section{Acknowledgements}

I would like to thank the organisers, especially Yoshinori Haga of JAEA, for my travel support and being able to attend the Conference in Sendai. 


\section{References}

[1] J.H. Shim, K. Haule and G. Kotliar, Fluctuating valence in a correlated solid and the anomalous properties of $\delta$-plutonium, Nature 446 (2007), pp. 513-516.

[2] M. Janoschek, P. Das, B. Chakrabarti, D. L. Abernathy, M. D. Lumsden, J. M. Lawrence, J. D. Thompson, G. H. Lander, J. N. Mitchell, S. Richmond, M. Ramos, F. Trouw, J.-X. Zhu, K. Haule, G. Kotliar and E. D. Bauer, The valence-fluctuating ground state of plutonium, $\mathrm{Sci}$. $A d v .10$ (2015), p. e1500188.

[3] B. Amadon, First-principles DFT+DMFT calculations of structural properties of actinides: Role of Hund's exchange, spin-orbit coupling, and crystal structure, Phys. Rev. B 94 (2016), pp. 115148-1-14.

[4] B. Dorado, F. Bottin, and J. Bouchet, Phonon spectra of plutonium at high temperatures, Phys. Rev. B 95 (2017), pp. 104303-1-5.

[5] P. Soderlind, Lattice dynamics and elasticity for E-plutonium, Sci. Repts. 7 (2017), pp. 1116-1-7.

[6] J.J. Joyce and G.H. Lander, Ch. 10 in the new "Handbook of Plutonium" - to be published, 2018

[7] A. Migliori, H. Ledbetter, A.C. Lawson, A.P. Ramirez, D.A. Miller, J.B. Betts, M. Ramos and J.C. Lashley, Unexpected elastic softening in $\delta$-plutonium, Phys. Rev. B 73 (2006), p.052101.

[8] K. Gofryk, S. Du, C.R. Stanek, J.C. Lashley, X.-Y. Liu, R.K. Schulze, J.L. Smith, D.J. Safarik, D.D. Byler, K.J. McClellan, B.P. Uberuaga, B.L. Scott and D.A. Andersson, Anisotropic thermal conductivity in uranium dioxide, Nat. Commun. 5 (2014), pp. 4551-1-7.

[9] P. Santini, S. Carretta, G. Amoretti, R. Caciuffo, N. Magnani and G.H. Lander, Multipolar interactions in f-electron systems: The paradigm of actinide dioxides, Rev. Mod. Phys. 81 (2009), pp. 807-863.

[10]C.H. Booth, Yu Jiang, D.L. Wang, J.N. Mitchell, P.H. Tobash, E.D. Bauer, M.A. Wall, P.G. Allen, D. Sokaras, D. Nordlund, T.-C. Weng, M.A. Torrez and J.L. Sarrao, Multiconfigurational nature of $5 \mathrm{f}$ orbitals in uranium and plutonium intermetallics, Proc. Natl. Acad. (USA) 109 (2012), p. 10205

[11]K.O. Kvashnina, H.C. Walker, N. Magnani, G.H. Lander and R. Caciuffo, Resonant x-ray spectroscopy of uranium intermetallics at the M4,5 edges of uranium, Phys. Rev. B 95 (2017), p. 245103

[12]T. Vitova, I. Pidchenko, D. Fellhauer, P.S. Bagus, Y. Joly, T. Pruessmann, S. Bahl, E. Gonzalez-Robles, J. Rothe, M. Altmaier, M.A. Denecke and H. Geckeis, The role of the $5 \mathrm{f}$ valence orbitals of early actinides in chemical bonding, Nat. Commun. 8 (2017), pp. 16053-1-9.

[13]G. Fabbris, D. Meyers, L. Xu, V.M. Katukuri, L. Hozoi, X. Liu, Z.-Y. Chen, J. Okamoto, T. Schmitt, A. Uldry, B. Delley, G.D. Gu, D. Prabhakaran, A.T. Boothroyd, J. van den Brink, D.J. Huang and M.P.M. Dean, Doping Dependence of Collective Spin and Orbital Excitations in the Spin-1 Quantum Antiferromagnet La2-xSrxNiO4 Observed by X Rays, Phys. Rev. Lett. 118 (2017), p.156402

[14]T. Gouder, L. Havela, F. Wastin and J. Rebizant, Evidence for the $5 \mathrm{f}$ localisation in thin Pu layers, Eur. Phys. Lett. 55 (2001), p.705

[15]A.K. Burrell, T.M. McCleskey, P. Shukla, H. Wang, T. Durakiewicz, D.P. Moore, C.G. Olson, J.J. Joyce and Q. Jia, Controlling Oxidation States in Uranium Oxides through Epitaxial Stabilization, Adv. Mater. 19 (2007), p.3559

[16]J.L. Sarrao, Y. Haga and R.C.C. Ward, New surprises "down below": Recent successes in the synthesis of actinide materials, MRS Bull. 35 (2010), p.877.

[17]R. Springell, R.C.C. Ward, J. Bouchet, J. Chivall, D. Wermeille, P.S. Normile, S. Langridge, S.W. Zochowski and G.H. Lander, Malleability of uranium: Manipulating the charge-density wave in epitaxial films, Phys. Rev. B 89 (2014), p.245101.

[18]S. Heathman, R.G. Haire, T. Le Bihan, A. Lindbaum, M. Idiri, P. Normile, S. Li, R. Ahuja, B. Johansson and G.H. Lander, A High-Pressure Structure in Curium Linked to Magnetism, Science 309 (2005), p.110. 